\title{
A Review on Cloud Computing Architectures
}

\author{
Sreelakshmi P. S. \\ College of Engineering \\ Trivandrum
}

\author{
Sabitha S. \\ College of Engineering \\ Trivandrum
}

\begin{abstract}
Cloud computing has emerged to meet the requirements of large, Internet based and data intensive applications. Commonly clouds are implemented on large data centers consisting of thousands of servers. All application processing and resources are centralized in these data centers. As the number of users increases, this centralized approach produce bottlenecks and affect the quality of cloud services. This brings inconvenience to users. Fortunately centralized approach is not the only way to provide cloud services. Other possible architectures for cloud computing are federated approach and peer to peer approach. Federated cloud combines independent clouds and provides interoperability between them. P2P clouds are a low cost model for cloud computing. This paper reviews centralized, federated and peer to peer approaches to cloud computing.
\end{abstract}

\section{Keywords}

Centralized Cloud, Federated Cloud, Peer to Peer Cloud

\section{INTRODUCTION}

Cloud computing is based on principles and technologies of distributed computing and virtualization. According to Mell and Grance [1], Cloud Computing is a model for enabling convenient, on-demand network access to a shared pool of configurable computing resources such as networks, servers, storage, applications, and services that can be rapidly provisioned and released with minimal management effort or service provider interaction. Clouds are capable of provisioning computing resources such as CPU time, network storage etc dynamically to users without human intervention. Cloud services can be accessed through network by thin or thick clients using standard mechanisms. Cloud service providers pool and assign virtual and physical resources dynamically to customers according to their demand. Cloud enable a customer application to scale out and scale in quickly. More resources can be provisioned quickly to customers as needed. If not needed resources can be dynamically released. Customer perceives unlimited resources that can be purchased at any time in any quantity. Cloud uses a pay per use model. The resource usages are monitored and reported to both customers and providers.

The interface between a cloud customer and a Cloud Computing environment is defined abstractly in service models. The different service models of Cloud Computing are Software as a Service, Platform as a Service and Infrastructure as a Service. In SaaS cloud, the cloud customer can access applications running on Cloud Infrastructure user web browser. A PaaS cloud provides capabilities such as programming languages, tools and hosting environment for ap-

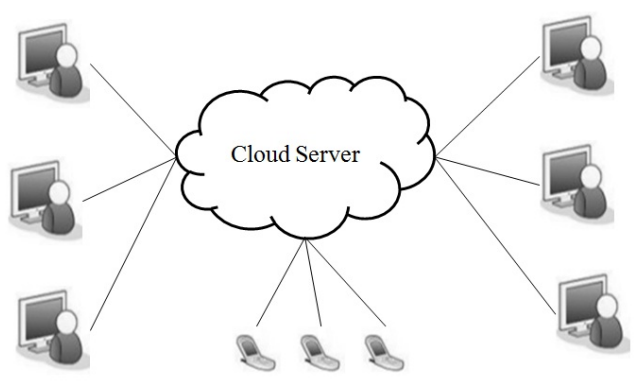

Fig. 1: Centralized Cloud Architecture

plications developed by the cloud customer. An IaaS Cloud can provide fundamental computing capabilities such as processing, storage and networks.

The development of Internet based, data intensive applications such as e-commerce and social networking, and the systems supporting these applications require a lot of computing and communication resources. The Cloud Computing can fulfill this demand. However, Centralized Clouds may cause performance bottlenecks if the number of customers increase tremendously. The use of multiple, interoperating Clouds become a necessity, to avoid the possibility of a single point of failure. Reservoir, Open Cirrus, InterCloud and contrail federates clouds to avoid single point of failure and to provide increased availability. Symfor [2], Wuala [3] and Spotify [4] uses peer to peer approach for providing cloud services at low cost. The Rest of this paper is organized as follows: Section 2, 3 and 4 discusses construction, advantages, limitations, application areas and challenges of centralized, federated and peer to peer approaches respectively. Section 5 concludes this paper with a general comparison of these approaches.

\section{CENTRALIZED CLOUDS}

A centralized cloud is implemented on large data centers consisting of thousands of servers. All application processing and resources are centralized in these data centers [5]. They are owned and operated by a single organization. A centralized cloud architecture is shown in figure 1 . Centralized clouds constitute the current commercial systems. Examples are Amazon EC2, Google App Engine, Windows Azure etc. 


\subsection{Advantages}

Business organizations can avoid in-house server storage and application requirements by using central clouds. The lack of onpremises infrastructure removes their associated operational costs such as power, air conditioning and administration costs. They are more reliable and consistent than in-house IT infrastructure. It simplifies IT management and maintenance capabilities through central administration of resources and vendor managed infrastructures.

\subsection{Limitations and Challenges}

Centralized clouds require huge investment for creation and maintenance. They are complex to manage due to large size. Here services and implemented on central servers. Server breakdown can cause service interruptions. This can cause serious troubles on critical aplications. Compute and storage services are offered on a best effort basis, ignoring problems such as QoS provisioning and Service Level Agreement(SLA) negotiations.

The most important concern in cloud computing is security and privacy. Essential services are outsourced to a third party. This makes it hard to maintain data integrity and privacy and support data and service availability. One of the biggest factors that the enterprises cite as a reason for not moving their business applications to cloud is service quality. SLAs provided by the cloud providers today are not sufficient to guarantee the requirements for running a production application that require high availability, performance and scalability. Without proper service quality guarantee enterprises are not going to host their business critical applications in the cloud. One of the major advantage of centralized approach is central management and administration. But it introduces single point of failure, which is a serious concern. Next section discusses cloud federations which is introduced as a solution to this problem.

\section{FEDERATED CLOUDS}

Federated clouds are formed by the integration of separate, independently owned and administered Clouds. They are also referred as "cloud of clouds" or "InterCloud" [7]. A cloud federation can be formed under the following circumstances: The cloud runs out of its resources. It can continue providing services to its clients, by renting resources from other clouds. The cloud needs to deploy a distributed cloud-based service in different geographical locations. It acquires resources placed in those target locations from other Cloud Service Providers. The cloud can federate with other Clouds for service relocation, power saving, backup etc.

\subsection{Federated Cloud Architectures}

There are three possible approaches for creating a federated cloud. They are Centralized Approach, Decentralized Approach and Hierarchical Approach. In centralized approach a cloud forms federation with other clouds through a third party broker. Broker is responsible for establishing connections between cloud requiring federation and those offering federations.

In decentralized approach clouds themselves manage discovery, communication, negotiation of agreements and matching and selection of the best partners for federation.

In hierarchical approach, Clouds are connected to a broker. Each Broker interacts with other brokers to look for clouds whose requirements match the requirements of clouds requesting partnerships. These three architectures are shown in figure 2

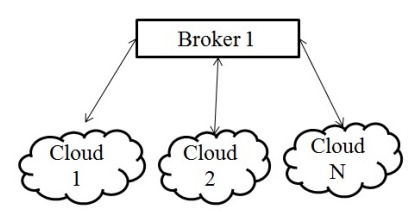

Centralized Approach

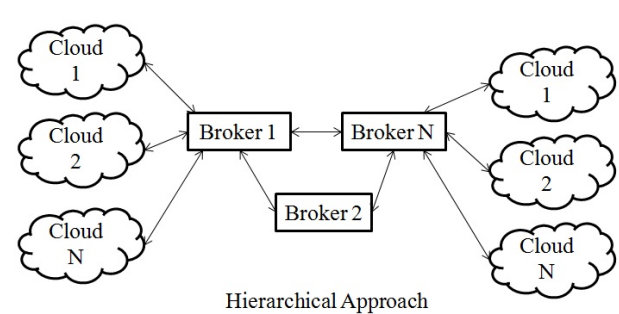

Fig. 2: Federated Cloud Architectures 6

\subsection{Advantages}

Federated Clouds have the following advantages :

-Cost Effective : Federated Clouds can provide a large amount of resources. This reduces completion time of a service, increases system throughput and optimize resource utilization.

-Effective Resource Utilization : A cloud service provider can provide resources to other clouds when it is not utilized

- Guaranteed Performance : Sudden increase in workload may decline performance of a single cloud service provider. Federated cloud can provide agreed QoS by renting resources from other Cloud Service providers.

- High Availability : Performance issues due to unexpected disasters can be cope up by federating with cloud service providers of unaffected areas.

-Avoid vendor Lock-in : If users are not satisfied with a cloud migration to other clouds is easy.

\subsection{Limitations and Challenges}

In centralized federations resource discovery is done by a third party. It lacks scalability and fault tolerence. In this approach if the broker fails, service become unavailable. Decentralized approach is complex than centralized approach. It also requires new methods for enhancing scalability and reliability.

Federated clouds should carry out system management without human intervention and manual administration. Therefore a cloud federation must be capable of doing automatically configuration of components, discovery and correction of faults, optimization of resource allocation and system security and integrity. Each cloud has its own methods and interfaces for providing various services. In federated clouds interoperability between different clouds is required. It should provide interfaces for communication between heterogeneous clouds. Similarly each cloud uses different authentication and identity management techniques. Identity management between clouds is needed to perform authentication among heterogeneous clouds. A global SLA management is required in federated clouds. Efficient techniques are required for discovery and provisioning of resources. 


\subsection{Applications}

Federated Clouds are appropriate for Multimedia entertainment [6]. For example, in the case of Massive Multiplayer Online Games (MMOGs), a large number of users interact in a virtual space. They require high Quality of Service. Multiple MMOG servers are being operated on geographically distributed Clouds to automatically balance the load. A cloud federation can be formed between these clouds. This helps to maintain a coherent game state.

Buyya et al. [7] presented a service-oriented architectural framework for federation of Cloud Computing environments. The goal of this framework is facilitating just-in- time, scalable provisioning of application services and consistently achieving quality of service targets under variable workload, resource and network conditions. The infrastructure described in this paper supports dynamic expansion or contraction of Virtual Machines, services, storage, and database for handling sudden variations in service demand and autonomic provisioning of QoS aware services across different Cloud providers while minimizing service costs.

Reservoir [8] is a European Union funded project. It aims to enable massive scale deployment and management of complex IT services across different administrative domains, IT platforms and geographies. The Reservoir project supports the emergence of federated clouds as a new computing paradigm. In this paradigm, services are software components made available through network accessible interfaces. It also enable the composition of complex distributed applications from loosely coupled components.

Extending the idea of federated clouds, P2P clouds are introduced. They are discussed in the following section.

\section{PEER TO PEER CLOUDS}

Peer to peer clouds or P2P Clouds are build by opportunistically assembling independent resources owned by individuals or organizations. This is a fully decentralized cloud architecture. They do not require any central monitoring or management components. Gossip based epidemic protocols can be used to monitor the Cloud, allocate resources and handle churn. P2P Clouds can provide services at low or zero cost. Loosely-coupled applications and distributed applications can be implemented on P2P CLouds [9].

\subsection{P2P Cloud Architecture}

Mei Xu et al. [10] proposed a cloud architecture by integrating P2P techniques into pure cloud. It is shown in figure 3. It consists of a core cloud and an extendable cloud. The core cloud based on a chord ring focuses on providing a reliable backup service such as a global resource index and replica storage. The chord ring mainly consists of nodes with high capability and good reliability.

The extensible cloud consists of huge amounts of ordinary nodes which frequently join or exit the system. They offer basic service such as data reading, writing and other frequent demanding from users. These nodes self organize into a hierarchical tree according to their capacity such as computing capability, online time, storage capacity and performance. A high-capability node can perform better than a low-capability node. Classifying nodes into different grades improve the performance of P2P cloud. If a node has a higher capacity and a better performance such as a lower accessing delay and a higher online ratio, its grade will be higher than others. Nodes are then self-organized into different clusters. Each cluster is composed of different grade nodes. To guarantee the reliability of the system, some first-grade nodes are selected to act as servers which can communicate information between lower-grade nodes and the core cloud, and also backup meta data such as directory

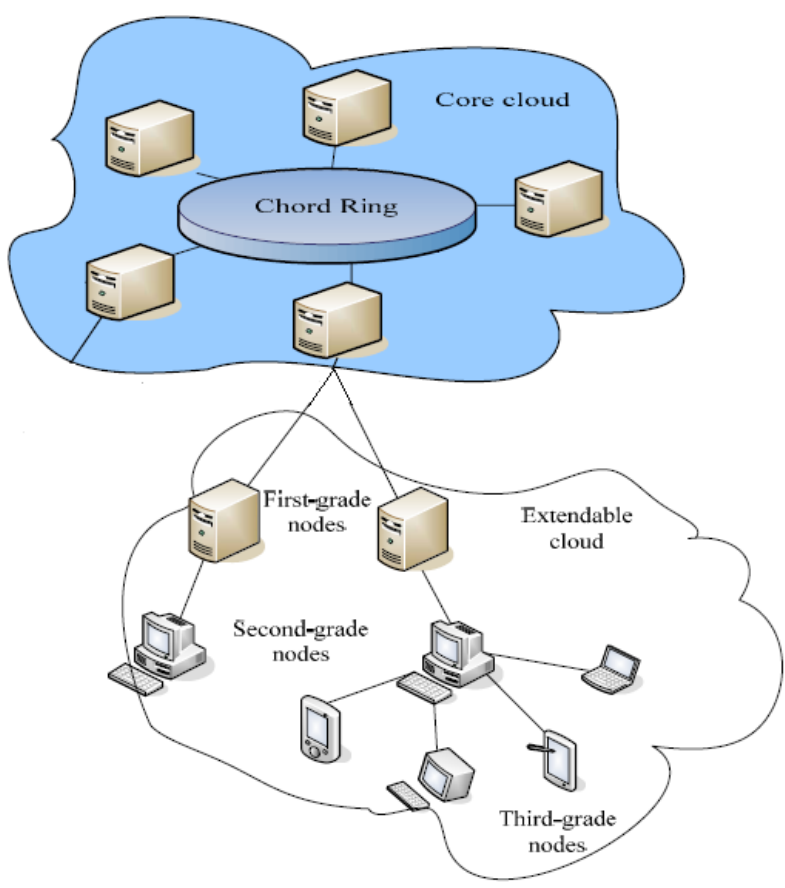

Fig. 3: Peer to Peer Cloud Architecture [10]

information, index of files and physical parameters of nodes for lower grade nodes and the core cloud. Once the first-grade servers fail, some second-grade nodes will automatically take the place of them. This infrastructure can achieve load balance and guarantee high QoS for Internet users by transferring load from the core cloud to the extendable cloud.

\subsection{Advantages}

P2P Clouds can provide on-demand scalability to customers. This approach does not introduces single point of failure. New resources can be added to or released from a P2P cloud easily. Peer to peer approach pools existing user resources to provide cloud services. Thus it can provide services at low cost.

\subsection{Limitations and Challenges}

Security and trust are extremely difficult to achieve in a fully distributed P2P Cloud scenario. Moreover, its expect to have a much lower Quality of Service than a centralized or federated Cloud. P2P paradigm is not yet appropriate for public clouds serving the general user community. However, a P2P cloud would be good for realizing a private infrastructure out of already existing resources owned by the same organization.

Fully decentralized peer to peer clouds is still an open research area. The BOINC project [11] introduced volunteer computing. It allows PC owners to contribute to a public resource pool, which then could be used by applications. This is not fully decentralized. It rely on centralized components. Maintaining the central components introduce a technical burden and a single point of failure. The current state of the art can be advanced by providing a system that can selfassemble and self-manage. Epidemic and gossip based protocols can be used for information dissemination and aggregation. Avoid- 


\begin{tabular}{|l|l|l|}
\hline Centralized & Federated & Peer to Peer \\
\hline $\begin{array}{l}\text { Single resource } \\
\text { provider }\end{array}$ & $\begin{array}{l}\text { Multiple resource } \\
\text { providers }\end{array}$ & $\begin{array}{l}\text { Multiple resource } \\
\text { providers }\end{array}$ \\
\hline $\begin{array}{l}\text { Virtualized environ- } \\
\text { ment }\end{array}$ & $\begin{array}{l}\text { Virtualized environ- } \\
\text { ment }\end{array}$ & $\begin{array}{l}\text { Virtualized environ- } \\
\text { ment }\end{array}$ \\
\hline High reliability & High reliability & $\begin{array}{l}\text { Unpredictable relia- } \\
\text { bility }\end{array}$ \\
\hline $\begin{array}{l}\text { Local or Geographic } \\
\text { Scale }\end{array}$ & Geographic Scale & $\begin{array}{l}\text { Local or Geographic } \\
\text { Scale }\end{array}$ \\
\hline Public or private & Public or private & Public or private \\
\hline
\end{tabular}

Table 1. : Comparison of Centralized, Federated and P2P approach to Cloud Computing

ing central services allows to further reduce the cost of deploying a P2P Cloud, making it a very low cost computing.

\subsection{Applications}

A P2P Cloud can be created at virtually no cost using existing resources. Therefore small or medium scale industries could turn idle resources into a cloud computing infrastructure which can then be allotted to internal users. For example, an engineering company could partition its spare desktop PCs among internal groups such as project team, IT group, accounting group etc. According to the needs of the various groups, more resources can be shifted towards them. Some applications which can be executed on a P2P Cloud include parallel computations, multimedia streaming, online gaming requiring low latency and a high level of interactively.

\section{CONCLUSION}

In this paper, we have discussed the advantages, disadvantages, challenges and application areas of three different cloud architectures namely Centralized Cloud, Federated Cloud and Peer to Peer Cloud. Single point of failure in centralized clouds is avoided in cloud federation. Peer to Peer approach can create low cost clouds. Fully decentralized P2P Clouds is still an open research area. Each of these architectures have different application areas in which it is more suitable. Peer to peer approach is suitable to set up a private cloud infrastructure. Centralized clouds are mostly public. Table 1 summarizes the architectures [10].

\section{REFERENCES}

[1] P. Mell and T. Grance, The NIST definition of cloud computing in NIST Special Publication 800-145, pp. 17, Sep. 2011.

[2] Symform web site(2016). [Online]. Available: http://symform.com/

[3] Ozalp Babaoglu, Moreno Marzolla, Michele Tamburini, Design and Implementation of a P2P Cloud System, in Universit di Bologna, Dipartimento di Scienze dellInformazione Mura A. Zamboni 7, I-40127 Bologna, Italy

[4] G. Kreitz and F. Niemela, Spotify - large scale, low latency, $P 2 P$ music-on-demand streaming, in Proc. 10th IEEE Int. Conf. Peer-toPeer Comput., 2010.

[5] F. Panzieri, O. Babaoglu, V. Ghini, S. Ferretti, and M. Marzolla, Distributed computing in the 21st century: Some aspects of cloud computing in Dependable and Historic Computing, volume 6875 of LNCS, 2011.

[6] Calcavecchia, N. Celesti, A. Nitto, Understanding Decentralized and Dynamic Brokerage in Federated Cloud Environments in M. Villari, I. Brandic, F.Tusa(Eds.), Achieving Federated and Self-Manageable Cloud Infrastructures: Theory and Practice, (pp. 36-56)

[7] Buyya R, Ranjan R, Calheiros RN, Intercloud: Utility-oriented federation of cloud computing environments for scaling of application services

[8] Rochwerger B, Galis A, Levy E, Caceres J, Breitgand D, Wolfsthal Y, Llorente I,Wusthoff M, Montero R, Reservoir: Management technologies and requirements for next generation service oriented infrastructures in Integrated Network Management, 2009.

[9] T. Mager, E. Biersack, and P. Michiardi, A measurement study of the Wuala on-line storage service, in Proc. 12th IEEE Int. Conf. Peer-to-Peer Comput., 2012, pp. 237248.

[10] Hai-Mei Xu, Yan-Jun Shi, Yu-Lin Liu, Fu-Bing Gao, Tao Wan, Integration of Cloud Computing and P2P: A Future Storage Infrastructure, 2012 IEEE

[11] Anderson DP, Boinc: A system for public-resource computing and storage, in Proceedings of the 5th IEEE/ACM International Workshop on Grid Comput- ing, IEEE Computer Society, Washington, DC, USA 